
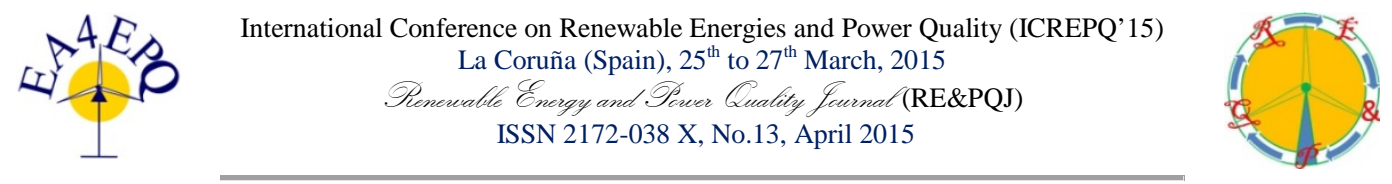

\title{
Towards a Better Understanding of Harmonics and Power Electronics - New Measurement Results and Analysis Methods for Modern Large-Scale Inverters
}

\author{
S. Rogalla, F. Ackermann, N. Bihler, G. Dötter, R. Singer, E. Wolf \\ Department Power Electronics \\ Fraunhofer Institute for Solar Energy Systems ISE \\ Heidenhofstr. 2, 79110 Freiburg (Germany) \\ Phone number: +49 (0) 761 4588-5454, e-mail: soenke.rogalla@ise.fraunhofer.de \\ http://www.ise.fraunhofer.de/en/business-areas/energy-efficient-power-electronics
}

\begin{abstract}
This paper deals with the harmonic current emission of large self-commutated PV inverters. It disproves with new measurement results that modern inverters behave as current sources for harmonic currents, which is the general idea behind the accepted grid codes. Furthermore it presents new approaches to quantify the contribution of grid voltage distortion to measured harmonic emission under real world conditions.

The conclusions which are drawn at the end of this paper are based on high precision harmonic current measurements, which have been carried out for a central inverter with a nominal power of $225 \mathrm{kVA}$. The harmonic emissions were measured for different types of grid configurations and background distortion.
\end{abstract}

\section{Key words}

Harmonic current emission, PV inverters, distorted grid voltage, harmonic current measurement.

\section{Introduction}

Loads as well as generators show in reality no pure sinusoidal grid currents. Harmonics cannot be fully avoided. In order to assure a reliable grid operation limits for harmonics are necessary. While harmonic emissions of grid commutated converters (diode rectifiers, thyristorbased inverters) are rather well-known [1], the harmonic behavior of modern self-commutating converter (e.g. pwm-controlled inverters) is still not fully understood.

With a simple experimental set-up it can be shown, that inverters do not behave as current sources: Assuming a current source behavior, the measured spectrums should not differ when changing the grid impedance. This assumption has already been disproved by measurements published in [2]. In [3] it was shown for a small-scale single-phase inverter that the harmonic content can be separated in to a power depending part and harmonics caused by grid voltage distortion.

Since large inverters are typically tested at non-ideal, distorted grids, the measured harmonic emission is usually higher than it should be for measurements at perfect sinusoidal grids.

\section{Experimental Setup}

The measurements, which are presented in this paper, were carried out in the 'Megawatt Laboratory' at Fraunhofer ISE in Freiburg, Germany.

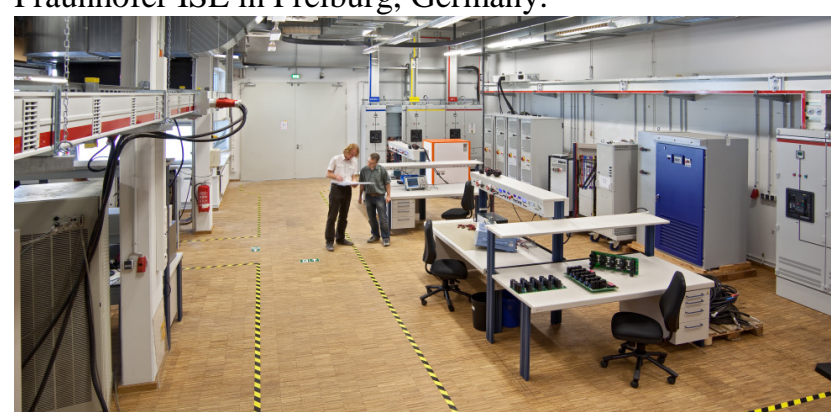

Fig. 1: Megawatt Laboratory at Fraunhofer ISE for testing inverters up to 1 MVA [4].

A. Device Under Test (DUT) and Testing Environment The device under test (DUT) was a PV inverter with a power rating of $225 \mathrm{kVA}$. The measurement setup is shown in Fig. 2. The DUT feeds via a transformer into the $20-\mathrm{kV}$-grid. The effective grid impedance can be varied strongly by adding a large line impedance (cf. Table I). The total grid impedance, which is effective for the DUT, sums up to $75 \Omega$, which results in a short circuit power of approx. 5.3 MVA. Hence, the resulting short circuit ratio referred to the nominal power of the DUT is approx. 23.5. This is a typical value for utility-scale PV power plants.

Table I. Effective grid impedances

\begin{tabular}{|l|c|c|}
\hline & $\begin{array}{c}\text { Impedance } \\
@ 20 \mathrm{kV}\end{array}$ & Type \\
\hline Public grid & $2 \Omega$ & Inductive \\
\hline Add. line impedance & $60 \Omega$ & Inductive \\
\hline Transformer sc-impedance & $13 \Omega$ & Inductive \\
\hline Total effective impedance & $\mathbf{7 5} \Omega$ & Inductive \\
\hline
\end{tabular}

In parallel to the DUT and its feed-in transformer a strong non-linear load (six-pulse rectifier with $65 \mathrm{~kW}$ ) can be 
connected as an additional “distortion generator". This leads to a harmonic distortion of the voltage at the terminals of the DUT.

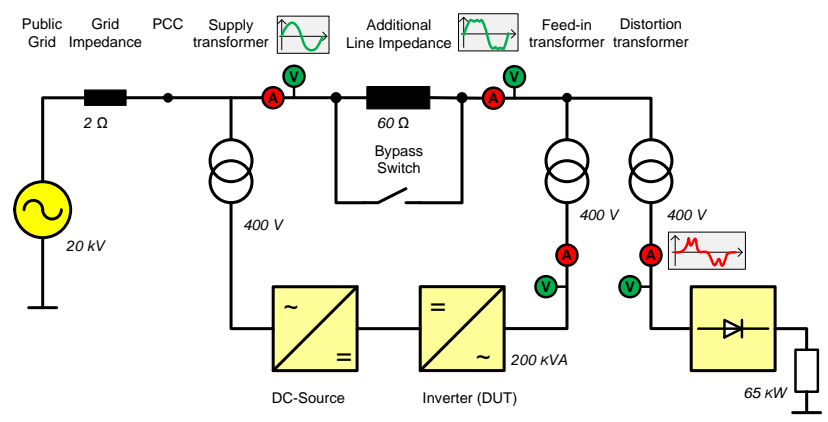

Fig. 2 Experimental setup. The voltage can be distorted by a sixpulse rectifier with a connected $65 \mathrm{~kW}$ load.

Voltages and currents were measured at the terminals of the DUT with a sampling frequency of $100 \mathrm{kHz}$. A DEWE-800 power meter with calibrated channels was used in combination with high precision close-loop current-compensated current transducers.

\section{B. Measurement Analysis}

For each point of operation ten-minute intervals of the voltages and currents at the terminals were recorded according to IEC 61000-4-7 [5]. A DFT-analysis with a spectral resolution of $5 \mathrm{~Hz}$ (200 ms windows) was carried out, resulting in 3.000 sets of spectra per ten-minute interval. Special attention was carried out in order to keep the phase information of the single harmonics during DFT-analysis. The results shown in the following were derived by aggregating all 3.000 sets. For the analysis of phase angle behavior spectral values with a $5 \mathrm{~Hz}$ resolution are used. Harmonics, inter-harmonics and higher frequency components have been calculated by grouping the spectrum accordingly.

\section{Influence of background distortion}

In this section measurements for different harmonic background distortion are analyzed. In initial condition the voltage at the DUT's terminals show a rather low background distortion level (THDU $=1.2 \%$ ). By means of the additional distortion generator (six-pulse rectifier) the voltage background distortion could be raised significantly (THDU $=3.0 \%$ ).

\section{A. Harmonics}

Fig. 3 shows the integer harmonics up to $2000 \mathrm{~Hz}\left(40^{\text {th }}\right.$ order) at nominal power, while Fig. 4 shows the interharmonics for the same conditions.

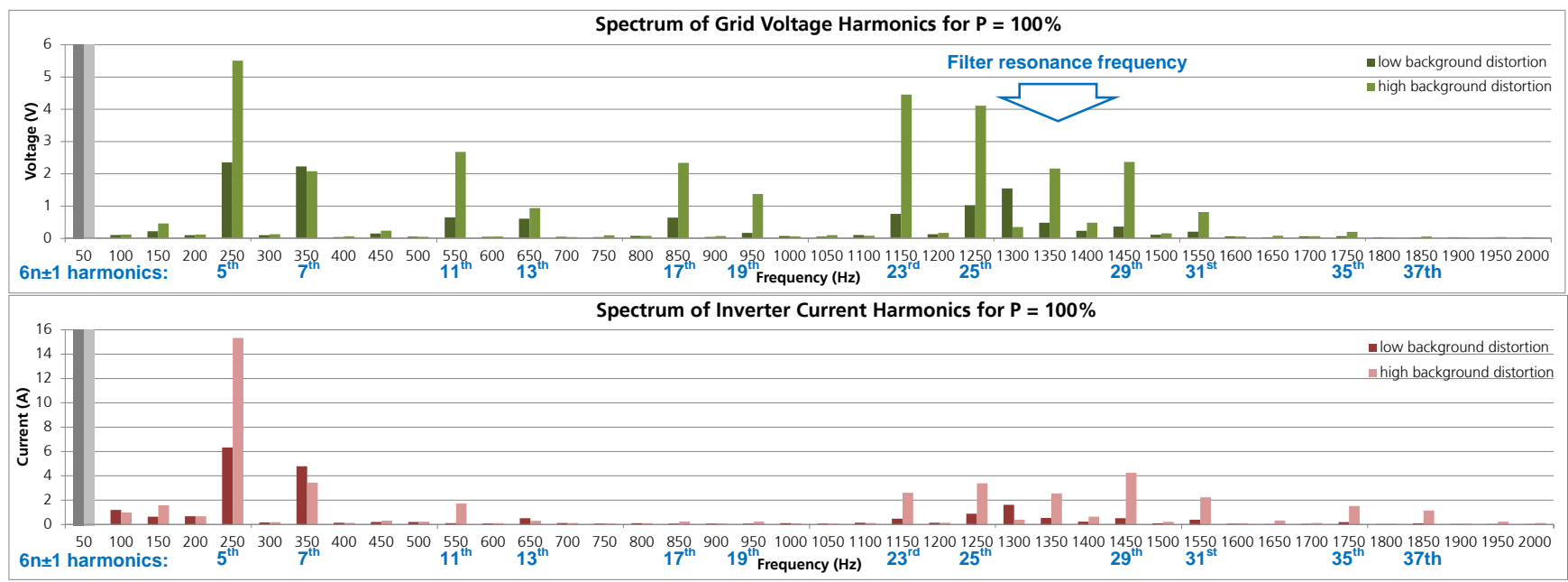

Fig. 3 Voltage and current harmonics with and without background distortion under nominal power $\left(\mathrm{P}_{\text {inverter }}=100 \% \mathrm{Pn}\right)$.
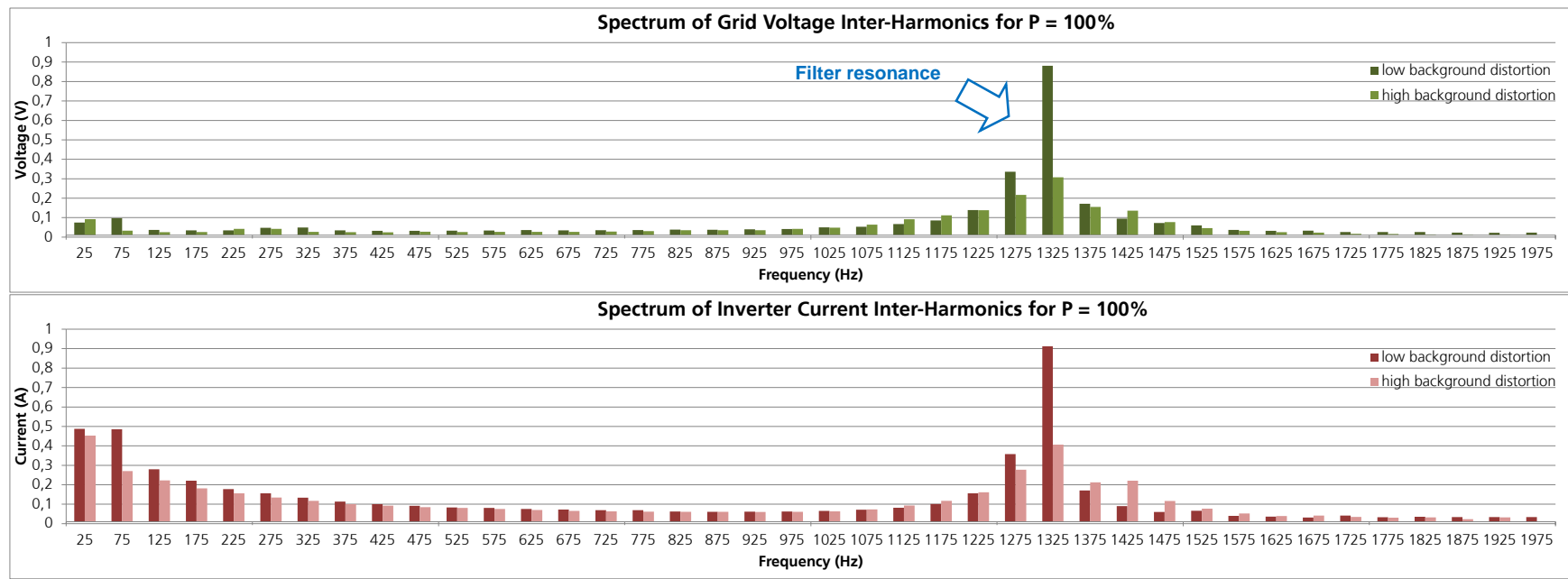

Fig. 4 Voltage and current inter-harmonics with and without background distorted for operation under nominal power (P = 100\% Pn). 


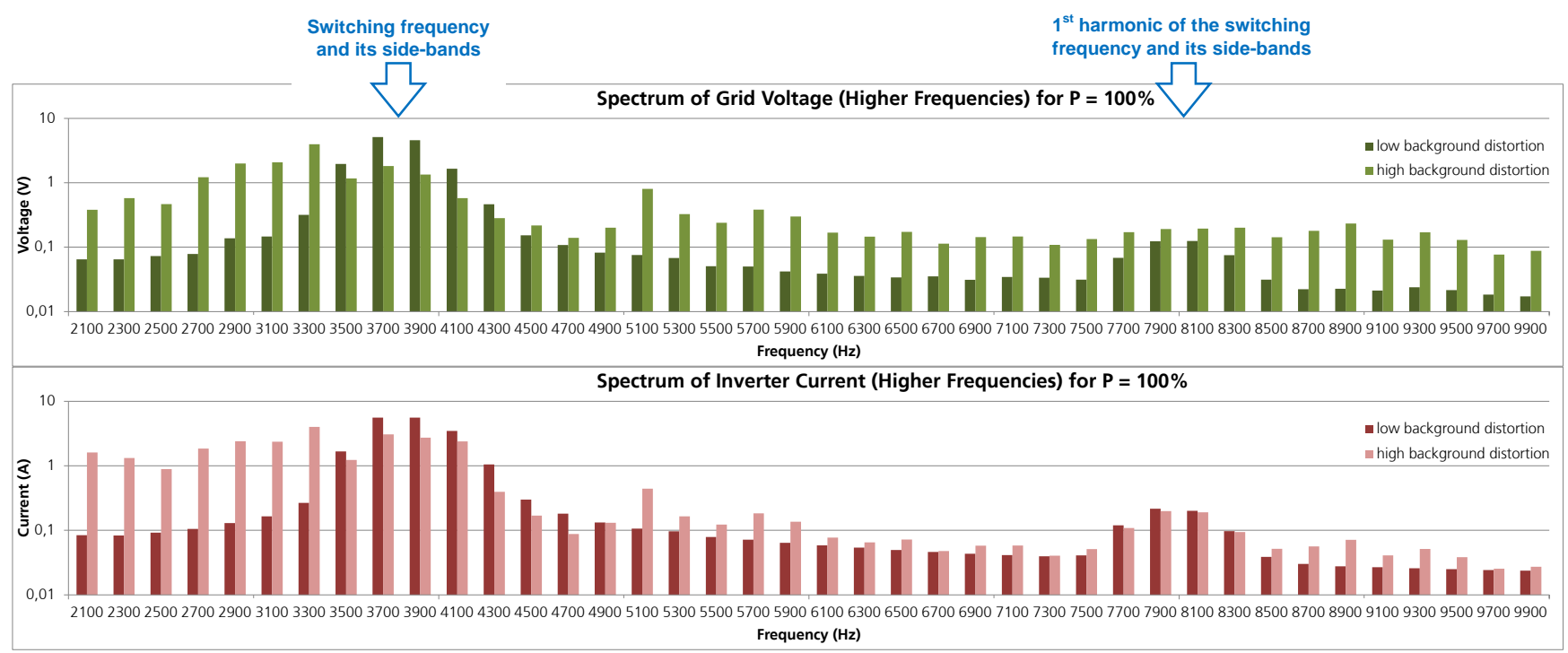

Fig. 5: Higher frequency components (2 - $10 \mathrm{kHz})$ of the voltage and current with and without background distorted for operation under nominal power $(\mathrm{P}=100 \% \mathrm{Pn})$.

From analyzing the spectra the following effects can be observed.

1) $6 \cdot n \pm 1$ harmonics. The harmonics current emission for the typical grid harmonics, which appear for orders given by $6 \cdot n \pm 1 \quad\left(5^{\text {th }} / 7^{\text {th }}, 9^{\text {th }} 11^{\text {th }}\right.$ ...) are increased significantly in case of higher background distortion. This is true except for the $7^{\text {th }}$ harmonics, where a slight decrease can be recognized. This might be explained with the fact, that the $7^{\text {th }}$ harmonic is already high in the reference situation with low overall background distortion. The distortion generator seems to have no an additional effect on this frequency.

2) Filter resonance $(1150-1550 \mathrm{~Hz})$. In the spectrum of the inter-harmonics the resonance frequency resulting from the inverters output filter (LCL-filter) together with the transformer and grid impedance is dominant in the frequency range from $1100 \mathrm{~Hz}$ to $1600 \mathrm{~Hz}$. Interestingly the inter-harmonics are decreased in case of higher background distortion. This can be explained by a damping and detuning effect caused by the parallel added 6-puls-rectifier. This non-linear load also leads to a shift of the resonance frequency, which can be observed by looking at the $26^{\text {th }}(1300 \mathrm{~Hz})$ and $27^{\text {th }}(1350 \mathrm{~Hz})$ harmonics. While the $26^{\text {th }}$ is reduced, the $27^{\text {th }}$ is increased. This means that the resonance is shifted to higher values.

\section{B. Higher frequency components}

Fig. 5 shows the spectrum of the higher frequency components (hf-component) in the range from $2 \mathrm{kHz}$ to $10 \mathrm{kHz}$ in logarithmic scale. Each hf-component covers a bandwidth of $200 \mathrm{~Hz}$. It is defined according to [5] as the square root of the sum of the squared $5-\mathrm{Hz}$ values in a range between $-95 \mathrm{~Hz}$ to $+100 \mathrm{~Hz}$ around the dedicated frequency.

1) Switching frequency. The switching frequency and its side-bands lead to high values in a spectrum around $4 \mathrm{kHz}$. The same repeats for the $1^{\text {st }}$ harmonic of the switching frequency around $8 \mathrm{kHz}$.

In case of high background distortions the hfcomponents in a range around the switching frequency is strongly reduced. Somehow the parallel added 6-pulse-rectifier seems to have a filtering influence on the spectrum of the voltage and current harmonics.

2) $6 \cdot n \pm 1$ harmonics. In case of a higher background distortion the hf-components increased significantly. This can be explained by the higher content of the $6 \cdot n \pm 1$ harmonics caused by the parallel added 6-pulse-rectifiers, which are present in this frequency range, too. The amount of increase depends on the number of $6 \cdot n \pm 1$ harmonics, which are included in the according $200 \mathrm{~Hz}$ band. The number differs between one and two.

\section{Conclusions}

Finally it can be noted, that a specific current harmonic is increased (reduced), if the corresponding voltage harmonic is increased (reduced) by an additional source of voltage distortion.

As a conclusion one can say, that the harmonic current emission of the DUT strongly depends on the background distortion of the grid during the measurements. Therefore a self-commutated inverter does not behave as a harmonic current source for different grid conditions.

\section{Influence of inverter's output power}

In this section measurements for different inverter output power are analyzed. The results for conditions with low and high background distortions do not differ significantly. Therefore only results for low background distortion are displayed here.

Fig. 6 shows the dependency of the harmonic spectra on the different output powers of the inverter. In each 
diagram three spectra for the inverter's power points at $0 \%, 50 \%$ and $100 \%$ of its output power are given. In the upper two diagrams the voltage and current harmonics, in the lower-two diagrams the inter-harmonics are presented.

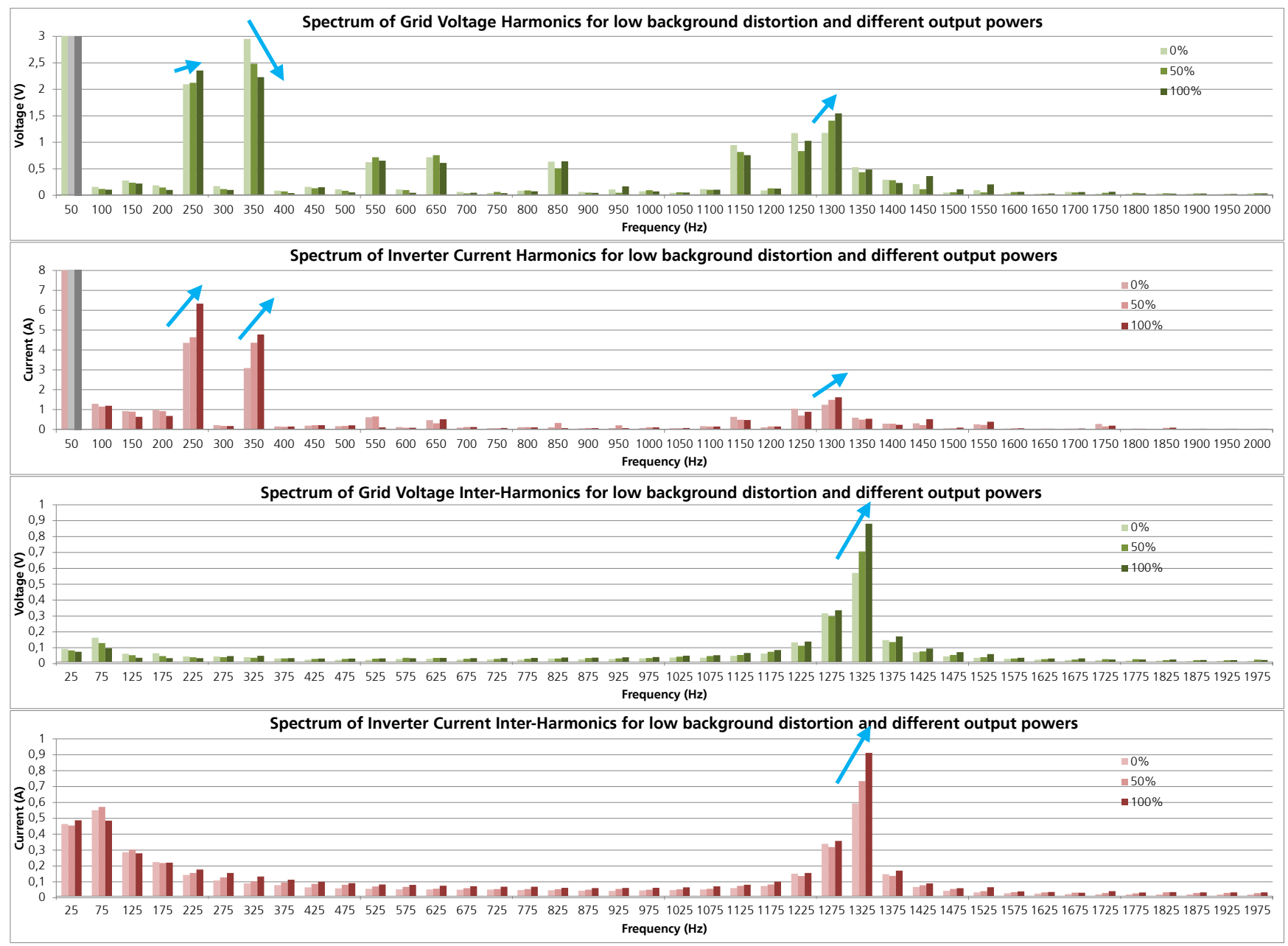

Fig. 6. Harmonics and inter-harmonics: Dependency of the voltage and current spectra depending on the inverter's output power under low background distortion conditions.

\section{A. Observed effects}

For the vast majority of harmonics the voltage as well as the current values raise, when the inverter power is increased (cf. the blue arrows in Fig. 6). This means that the inverter emits higher harmonic currents at higher output powers, which can provoke a higher grid voltage distortion.

But this behavior is not true for the $7^{\text {th }}$ harmonics $(350 \mathrm{~Hz})$. At this frequency a power dependent increasing current leads to a reduction of the according harmonic voltage. For this particular voltage the grid voltage is even improved by the inverter's behavior.

\section{B. Conclusions}

For the DUT it turns out, that typically a higher output power leads to a higher grid voltage distortion caused by the inverter. But this behavior cannot be generalized, as shown for the $7^{\text {th }}$ harmonic order.

Furthermore it must be pointed out, that the measurements presented in this paper had been performed for one grid impedance angle only so far. Therefore the conclusion cannot be generalized for any grid impedance situation for the moment.

\section{Complex values of the harmonics}

In this section not only the amplitude but also the prevailing phase angle of the particular harmonics is analyzed. This gives complex values of the voltage, current and power harmonics, which can be displayed in the complex plane.

Fig. 7 shows the scatter plots for the complex harmonic voltages, currents and impedances at following selected frequency.

- $250 \mathrm{~Hz}$ (5 $5^{\text {th }}$ harmonic)

- $350 \mathrm{~Hz}\left(7^{\text {th }}\right.$ harmonic)

- $1350 \mathrm{~Hz}$ (filter resonance frequency)

- $3900 \mathrm{~Hz}$ (switching frequency)

In each diagram point clouds for low (green) and high (red) background distortion are displayed. For both conditions the scatter plot contains the results for eleven measured power levels $(0 \%, 10 \%, \ldots, 100 \%)$. 

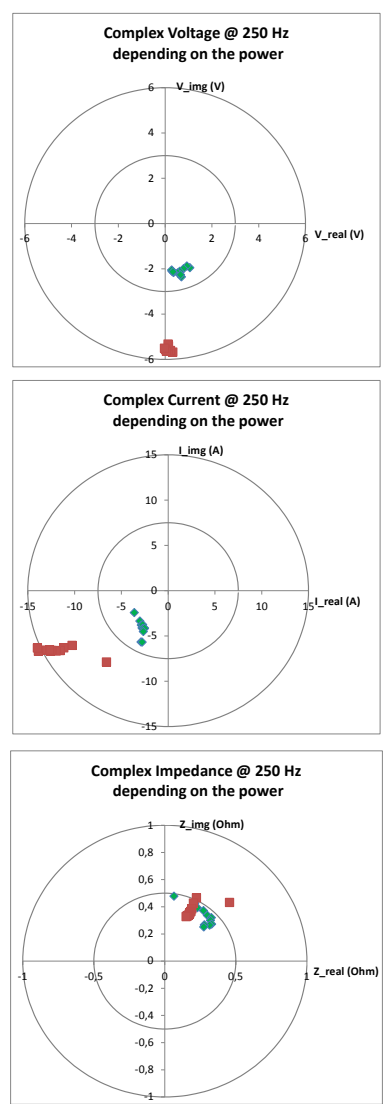
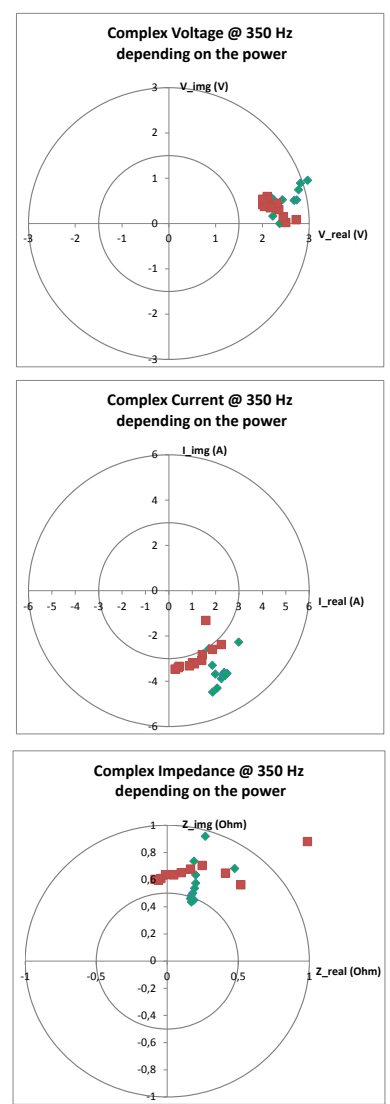
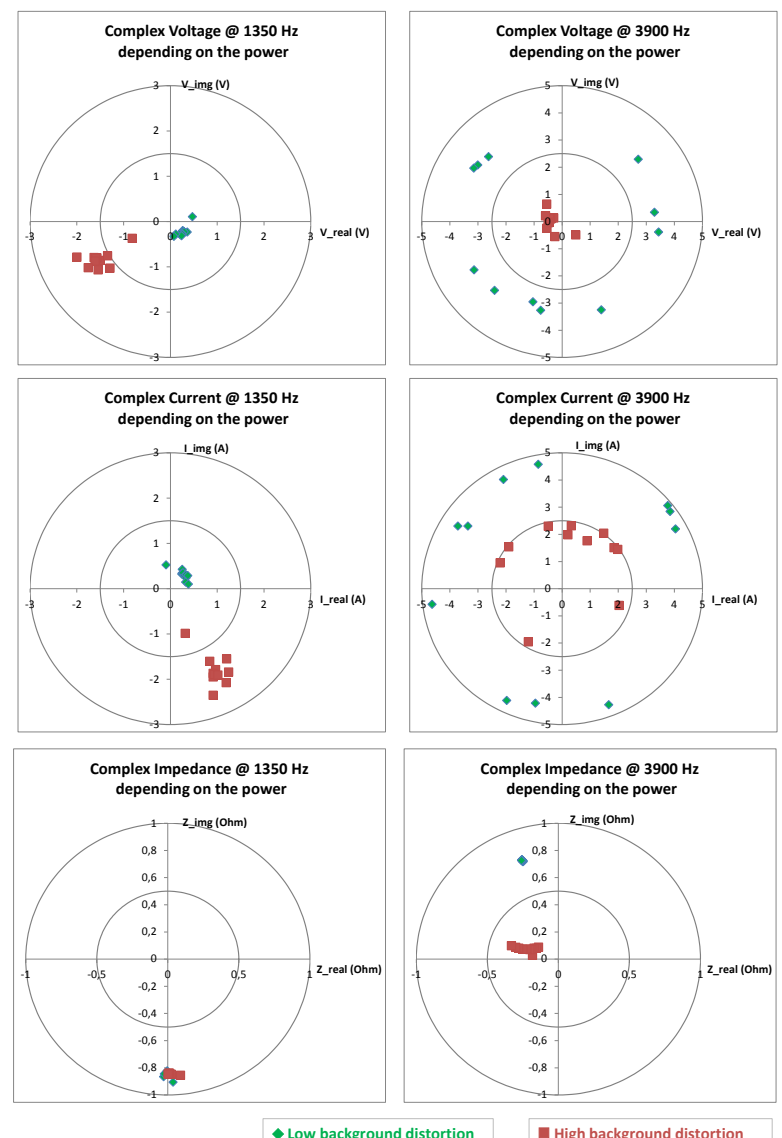

Fig. 7 Scatter plots of the complex values of voltages, currents and impedances for selected frequencies for different powers $(0 \%, 10 \%, \ldots$ $100 \%)$

The position of the voltage and current points give some indication about the phase shift referred to the phase of the fundamental frequency. It turns out that the phase is not significantly influenced by the background distortion.

For low harmonics $\left(5^{\text {th }}\right.$ and $7^{\text {th }}$ order) all measurement points appear highly concentrated, meaning, that the phases of the single harmonics show a fixed prevailing angle. This is due to the fact that these harmonics are synchronous to the fundamental frequency.

However, for typical PV-inverters the switching frequency is not synchronized to the fundamental frequency of the grid. Hence, voltage and currents do not have a fixed prevailing angle at the switching frequency. As a consequence the points in the diagrams for the voltage and current harmonics spread on a concentric circle, as can be regarded in the right-column diagrams.

The diagrams in the lower row of Fig. 7 show the complex harmonic impedance values $\boldsymbol{Z}_{\boldsymbol{v}}$, which had be calculated from the complex voltage harmonics $\underline{\boldsymbol{V}}_{\boldsymbol{v}}$ and the current harmonics $\underline{\boldsymbol{I}_{\boldsymbol{v}}}$ as follows.

$$
\underline{Z_{v}}=\frac{V_{v}}{\underline{I_{v}}}
$$

By analyzing the quadrant, in which the impedance is located, the character of the particular harmonic can be defined. With this method it can be determined whether a specific harmonic has a source (negative real part) or sink (positive real part) behavior. Furthermore it can be analyzed, if it behaves inductive (positive imaginary part) or capacitive (negative imaginary part).
It is interesting to see, that the prevailing angle of the complex impedance is quite stable, even if the prevailing angle of the voltage and current harmonics are not (as it is the case for $3900 \mathrm{~Hz}$ ).

It turns out, that the $5^{\text {th }}$ and $7^{\text {th }}$ order harmonics show an ohmic-inductive character. Assuming, that the grid impedance at this frequency is also ohmic-inductive, the inverter's current causes a voltage drop on the grid impedance, which reduces the according voltage harmonic at the point of common coupling. The inverter behaves grid voltage improving for this frequency.

In contrast the inverter behaves purely imaginary with capacitive character at the filter resonance frequency $(1350 \mathrm{~Hz})$. The inverter is equipped with an LCL-filter, which is in resonance with the grid impedance. The impedance of the output inductor is small compared with the impedance of the filter capacitor. Resistive parts can be neglected here. This explains the capacitive characteristic of the resulting inverter impedance. It should be mentioned, that this can be a critical issue for stable operation in case of systems with lacking damping.

For the switching frequency the inverter shows an inductive characteristic with some additional active power injection. In case of high background distortion the imaginary (inductive) part of reduces strongly, while the real (active) part is almost unaffected. Assuming the inverter behaves as a voltage source for switching frequency harmonics the only conclusion, which can be drawn from this result, is, that the parallel added 6-pulse- 
recifier changes the resulting total grid impedance in such a way, that the ohmic content is retained, while the inductive component is strongly reduced.

\section{Harmonics Power Injection and Power Prevailing Angel}

In order to analyze the characteristic of the harmonics of the full spectrum the power prevailing angle for all frequencies can be calculated. (Note: Analysis methods like prevailing angel ratio calculation are based on the methods, which are currently discussed in the IEC TC82 concerning the revision of the new IEC 61400-21 [6].)

Before deriving a conclusion from the spectrum of the power prevailing angle it makes sense to filter the values. Two filter steps had been applied here. In a $1^{\text {st }}$ step only frequencies with reasonable values of the harmonic power are considered. Fig. 8 shows the spectrum of the power harmonics with a threshold line. Only frequencies with values above this line are considered. The threshold $\boldsymbol{t}(\boldsymbol{f})$ was empirical defined to

$$
t(f)=\frac{k}{f^{\alpha}} \quad, \text { with } k=100 \text { and } \alpha=1.4
$$

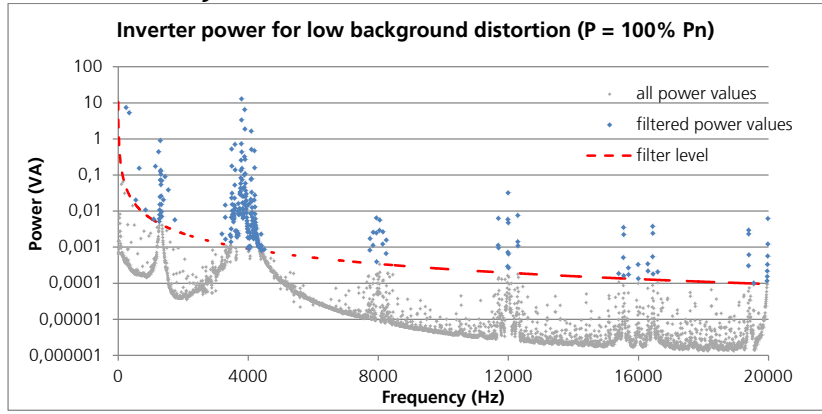

Fig. 8. Spectrums of the amplitudes of the inverter output power. For background distortions. Relatively high values are filtered and used for further considerations.

In a second step the power prevailing angle ratio (ppar) is filtered with a constant threshold value of 0.95 ; as it is shown in Fig. 9. The ppar takes all 3000 sets of spectra into account, which are generated within the ten-minute interval. The ppar evaluates the stability of the 3000 phase angles between Harmonic currents and the related Harmonic voltage. In case all of them are in phase that ppar value is unity. In case of a randomized distribution of the phase angles the value tends to zero. Mathematically the ppar is defined in as ratio between the geometrical sum and the sum of the absolute values of the 3000 complex power harmonic values.

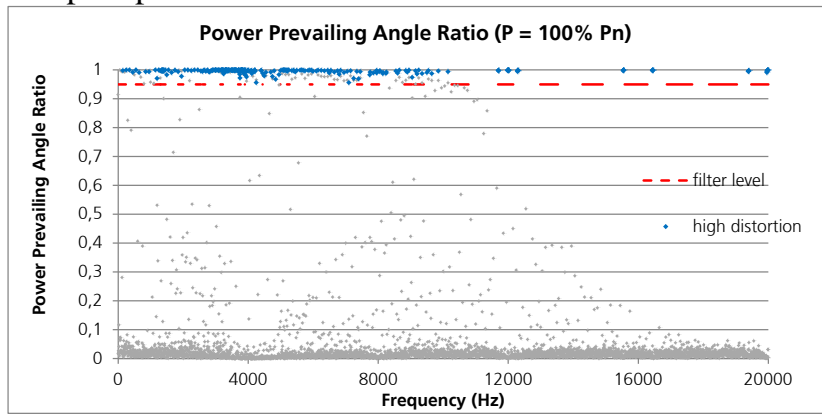

Fig. 9. Spectrums of the power prevailing angle ratio of the inverter output power. For high background distortions. Values are filtered and only high values $>0.95$ are used for further considerations.

In Fig. 10 the values for the ppar are displayed only for frequencies, which have passed the two filter steps described before. Therefore only values for frequencies with reasonable harmonic content und stable phase angles are shown.

It turns out that the majority of points concentrate either close to $0.5 \pi$ or $1.5 \pi$, meaning, the most harmonics show either inductive or capacitive behavior.

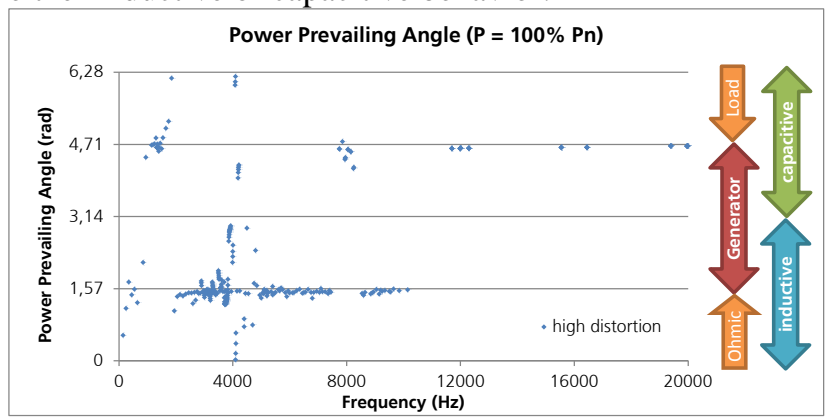

Fig. 10. Spectrums of prevailing angles for low and high background distortions.

\section{Conclusions}

The presented results help to improve the general understanding of the harmonic behavior for selfcommutated inverters. It could be shown with the presented results, which are based on real measurements of a $225 \mathrm{kVA}$ inverter, that the background distortion caused by a non-linear load strongly affects the harmonic current spectrum of a self-commutated PV-inverter. It was found out, that amplitude of the current spectrum strongly correlates with the particular voltage spectrum. By analyzing the influence of the inverter's output power, it could be shown that the harmonic current emission sometimes helps to improve the voltage quality, especially for low-order harmonics $\left(5^{\text {th }} / 7^{\text {th }}\right)$. By plotting the complex harmonic values we came to the conclusion, that the impedance of existing harmonics typically has a clear and stable phase angle regardless whether the harmonic currents or voltage are synchronized with the fundamental frequency. This type of analysis can help to determine the characteristic of single harmonics and therefore clarify their impact on the power quality.

\section{References}

[1] A. Baggini, Handbook of power Quality, Wiley (2008)

[2] Rogalla, S.; et. al., Schwierige Bewertung von Oberschwingungsemissionen, in Proc., 1. ZNE, 2014, Berlin, Germany, (29.-30.1.2014),

[3] Rogalla, S.; et. al., »Verbesserter Ansatz zur Bestimmung der Oberschwingungsemissionen von Zentralwechselrichtern«, in Proc., 27. SOL2012, Bad Staffelstein, Germany, (29.2.-2.3.2012)

[4] www.ise.fraunhofer.de/en/service-units/inverter-lab

[5] IEC 61000-4-7:2002 Electromagnetic compatibility (EMC) Part 4-7: Testing and measurement techniques, (2002)

[6] IEC CD 61400-21-1:2014: Wind turbines - Part 21-1: Measurement and assessment of electrical characteristics 